The Journal of Laryngology \& Otology

http://journals.cambridge.org/JLO

Additional services for The Journal of Laryngology \& Otology:

Email alerts: $\underline{\text { Click here }}$

Subscriptions: $\underline{\text { Click here }}$

Commercial reprints: Click here

Terms of use : $\underline{\text { Click here }}$

\title{
Dysphonia secondary to traumatic avulsion of the vocal fold in infants
}

D Bray, L Cavalli, N Eze, N Mills and B E J Hartley

The Journal of Laryngology \& Otology / Volume 124 / Issue 11 / November 2010, pp 1229 - 1233

DOI: 10.1017/S0022215110001131, Published online: 24 May 2010

Link to this article: http://journals.cambridge.org/abstract_S0022215110001131

How to cite this article:

D Bray, L Cavalli, N Eze, N Mills and B E J Hartley (2010). Dysphonia secondary to traumatic avulsion of the vocal fold in infants. The Journal of Laryngology \& Otology, 124, pp 1229-1233 doi:10.1017/S0022215110001131

Request Permissions : $\underline{\text { Click here }}$ 


\title{
Dysphonia secondary to traumatic avulsion of the vocal fold in infants
}

\author{
D Bray, L Cavalli*, N Eze, N Mills, B E J Hartley
}

\begin{abstract}
Objective: Airway compromise due to paediatric intubation injuries is well documented; however, intubation injuries may also cause severe voice disorders. We report our experience and review the world literature on the voice effects of traumatic paediatric intubation.

Case series: We report five cases of children referred to Great Ormond Street Hospital for Children who suffered traumatic avulsion of the vocal fold at the time of, or secondary to, endotracheal intubation. All children had significant dysphonia and underwent specialist voice therapy.

Conclusions: The mechanisms of injury, risk factors and management of the condition are discussed. Children suffering traumatic intubation require follow up throughout childhood and beyond puberty as their vocal needs and abilities change. At the time of writing, none of the reported patients had yet undergone reconstructive or medialisation surgery. However, regular specialist voice therapy evaluation is recommended for such patients, with consideration of phonosurgical techniques including injection laryngoplasty or thyroplasty.
\end{abstract}

Key words: Larynx; Child; Trauma; Voice; Vocal Cords

\section{Introduction}

In neonates, suction, intubation and instrumentation are all possible causes of vocal fold trauma. Post-intubation laryngeal dysfunction has been well documented in adults. The dangers of intubation begin with insertion of the tube. Immediate complications are well recognised, such as oropharyngeal abrasion, and lacerations of the oropharynx, hypopharynx and larynx. Cricoarytenoid joint fixation, granuloma formation and subglottic stenosis are relatively common longer term patterns of injury. Cricoarytenoid dislocation, arytenoid subluxation, oesophageal perforation, tracheal rupture and retropharyngeal abscess formation have all been reported. Other than a previous report of case four from this current paper, ${ }^{1}$ there have been no published descriptions of complete traumatic avulsion of the vocal fold secondary to intubation in the neonatal period. Traditionally, attention has been focused on the airway sequelae of paediatric intubation injuries; relatively little has been written on the detrimental effects to the voice.

\section{Case series}

Case one

A neonatal male underwent repair of a double outlet right ventricle and hypoplastic aortic arch on the second day of birth. He was subsequently ventilated for six months in a cardiac intensive care unit.

At six months, a tracheostomy was inserted to aid weaning from ventilation.

At one year, the boy was admitted for microlaryngoscopy, bronchoscopy and KTP-laser treatment to supra- stomal granulation tissue, with a view to decannulation. At operation, the supra-stomal granulation was lasered, and it was noted that the right vocal fold was absent (Figure 1). Distally, there was an area of malacia in the left main bronchus.

Until eight months of age, no vocalisation was heard due to the size of the tracheostomy tube. A speaking valve was successfully fitted at 10 months of age, after down-sizing of the tracheostomy tube. Development of voice was gradual. At 10 months, vocalisation was heard only when the child was excited or upset, and had a very high pitch, with a harsh, breathy quality and reduced loudness.

Early speech and language therapy intervention focused on Makaton signing and development of language skills. Vocal play was encouraged with songs and imitations of animal and environmental noises, with a focus on working through high pitches to encourage approximation of residual phonatory structures.

The boy was successfully decannulated at the age of 22 months.

By three years of age, he had only mild to moderate dysphonia characterised by a harsh, breathy quality, mildly reduced loudness and mildly raised habitual pitch with adequate pitch range. Voice production was accompanied by some laryngeal tension. Problems with being heard in nursery were managed through environmental changes.

When older, direct voice therapy may be appropriate for this patient. He will be monitored within the joint voice clinic throughout puberty, both with respect to vocal changes and to the psychosocial impact of his voice disorder.

From the Departments of Paediatric Otolaryngology and *Speech and Language Therapy, Great Ormond Street Hospital for Children, London, UK.

Accepted for publication: 19 February 2010. First published online 24 May 2010. 


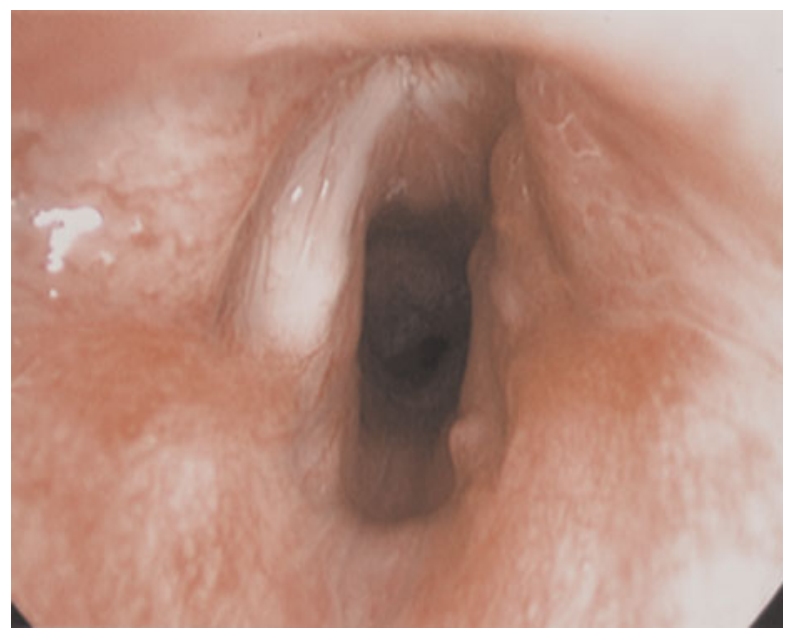

FIG. 1

Case one: Absent right vocal fold.

\section{Case two}

A male neonate was born prematurely at 24 weeks' gestation with severe tracheomalacia, and was intubated and ventilated for seven months, prior to undergoing tracheostomy. After tracheostomy insertion, he was weaned to continual positive airways pressure, which was continued until three years of age.

At three years, the subglottic stenosis resulting from the child's prolonged intubation was repaired by single stage laryngotracheal reconstruction.

He was successfully decannulated the following year.

At 10 years of age, stertor and nocturnal snoring prompted a sleep study, together with microlaryngoscopy and bronchoscopy. The sleep study confirmed moderate upper airways obstruction, which improved with subsequent adenotonsillectomy. At microlaryngoscopy and bronchoscopy, the right vocal fold was noted to be ragged, scarred and atrophic, and the right cricoarytenoid joint to be fixed (Figure 2).

Initial speech and language therapy assessment of this child's voice occurred at seven years of age. At that time, his voice was noted to be moderately to severely dysphonic, and characterised by a low loudness level, raised pitch, breathy quality with an inspiratory component. The patient used significant laryngeal and body tension to project his voice above background noise and across distances. Therapeutic aims were complicated by a conductive hearing loss and learning difficulties associated with prematurity: specifically, reduced memory recall and poor attention control. However, direct voice therapy intervention, offered at eight years, focused on improving breathing for voice, and a reduction was noted both in the use of inspiratory voice and the tension levels accompanying voice production. The child was also prescribed a voice amplification system to augment his vocal loudness in the noisy classroom environment.

The child came to our voice clinic at 10 years of age. Dynamic assessment with stroboscopy confirmed a large glottic chink on phonation, with approximation of the left vocal fold towards the right ventricular fold but limited contact. The left mucosal wave was reduced due to scar tissue. The possibility of a medialisation procedure postpuberty was discussed.

At the age of 15 years, the boy was active and involved in sports, but could not keep up with his peers and needed frequent rests. Frequent choking episodes, without subsequent lower respiratory tract infections,

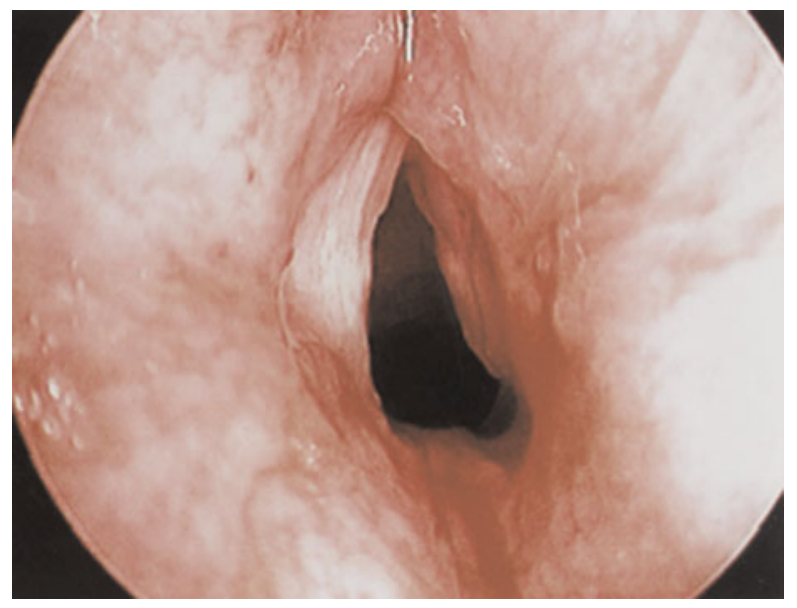

FIG. 2

Case two: Ragged, scarred and atrophic right vocal fold.

necessitated a videofluoroscopy, which showed deep laryngeal penetration without aspiration. Swallowing technique training, and diet modification with thickened fluids, were trialled. A computed tomography scan of the boy's chest confirmed features of bronchopulmonary dysplasia. His asthma was well controlled on medication.

At the time of writing, the boy's voice remained weak and he still snored loudly, so microlaryngoscopy and bronchoscopy were planned for the near future.

\section{Case three}

A 13-year-old girl was referred to our voice clinic with deterioration in voice quality and difficulty in producing a loud voice.

She had been born prematurely at 23 weeks' gestation, and had been intermittently intubated for the first three months of life. Microlaryngoscopy and bronchoscopy had shown granulations in the subglottis and a large laryngeal mucosal ulcer. She had been successfully extubated after her mild subglottic stenosis was managed with a cricoid split, albeit with some stridor in her early years.

At the age of 13 years, after 18 months of voice therapy at her local hospital, she was referred to us with ongoing difficulty in being heard in social contexts, and embarrassment regarding her voice.

Flexible nasendoscopy showed an absent left aryepiglottic fold and left vocal fold, with scar tissue. The significant scarring throughout the left side of the larynx had resulted in prolapse of the epiglottis to the left (Figure 3).

Perceptual voice evaluation suggested an inconsistent voice, with short episodes of supraglottic type vocalisation characterised by low pitch and harsh voice quality. The girl's habitual voice quality was breathy with some harshness exacerbated by fatigue or stress.

Laryngovideostroboscopy was performed in the voice clinic, which showed the girl's habitual voice to be produced with significant lateral and antero-posterior supraglottic compression. There was some passive prolapse of the epiglottis, and the right vocal fold moved normally in both abduction and adduction. However, the right fold failed to approximate against the left glottic scar band, and a glottic chink was seen along the entire vocal fold on phonation. Voice production was predominantly supraglottic.

This examination suggested that glottic closure may be possible following instruction to produce firm glottal onsets. Voice therapy deconstriction manoeuvres successfully relaxed the supraglottic tension. It would have been 


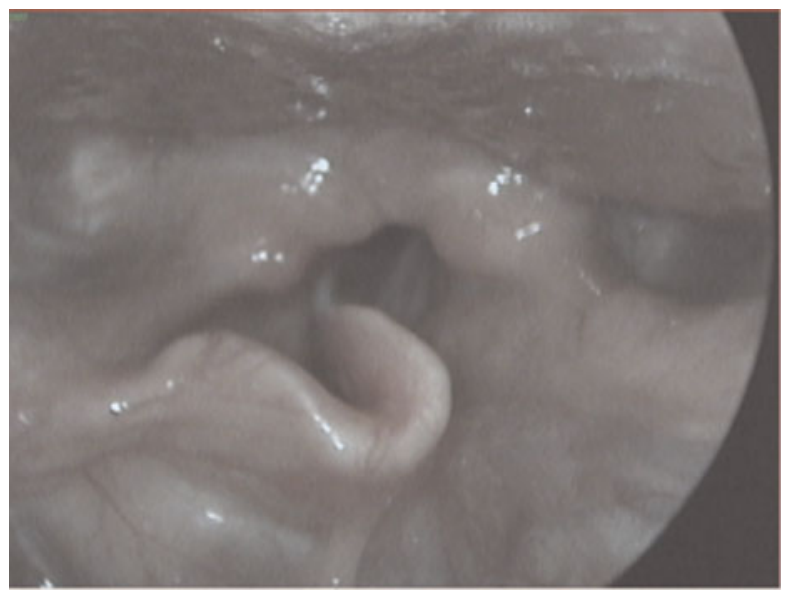

FIG. 3

Absent left aryepiglottic fold and left vocal fold. Scarring resulting in leftward laryngeal rotation

possible to augment the left vocal fold to improve the voice; however, in the presence of exercise intolerance secondary to mild subglottic stenosis, it was thought inappropriate to perform phonosurgery before puberty.

At the time of writing, the girl was undergoing voice therapy to facilitate reduction of the unhelpful laryngeal constriction, firmer glottal onsets, and improved vocal loudness. She will be considered for medialisation of the left vocal fold if her airway function normalises after pubertal laryngeal growth.

\section{Case four}

A four-year-old boy was referred to Great Ormond Street Hospital for Children for further management.

At two years of age, he had presented to a regional ENT department with dysphonia. He was a twin born at 28 weeks' gestation, and had required endotracheal intubation and ventilation for 18 days.

With the development of speech, it had been noted that the boy's voice was permanently hoarse, exacerbated by upper respiratory tract infections. He was not stridulous at rest and had a good cough. Direct laryngotracheobronchoscopy revealed an absent middle third of the right vocal fold (Figure 4)

In our voice clinic, dynamic flexible nasendoscopy confirmed the boy's phonatory source to be the normal left vocal fold vibrating against the residual right vocal fold mass and scar tissue, with minimal supraglottic involvement. His voice was moderately to severely dysphonic, with a low pitched, harsh voice quality and low loudness level. His voice was unstable, with pitch breaks in the upper pitch range and voice breaks during continuous speech. Breathing for speech was suboptimal. Surgical options were not thought appropriate, so voice therapy was recommended to improve vocal stability.

\section{Case five}

A five-month-old female infant presented with a weak cry and hoarse voice since birth.

She had been born prematurely at 26 weeks, and had been ventilated via an endotracheal tube for one month during the neonatal period. At six weeks, she had developed meningitis and cytomegalovirus infection, and had been intubated and ventilated again for a further 48 hours.

Other than bilateral sensorineural hearing loss, for which the child wore bilateral hearing aids, she was well.

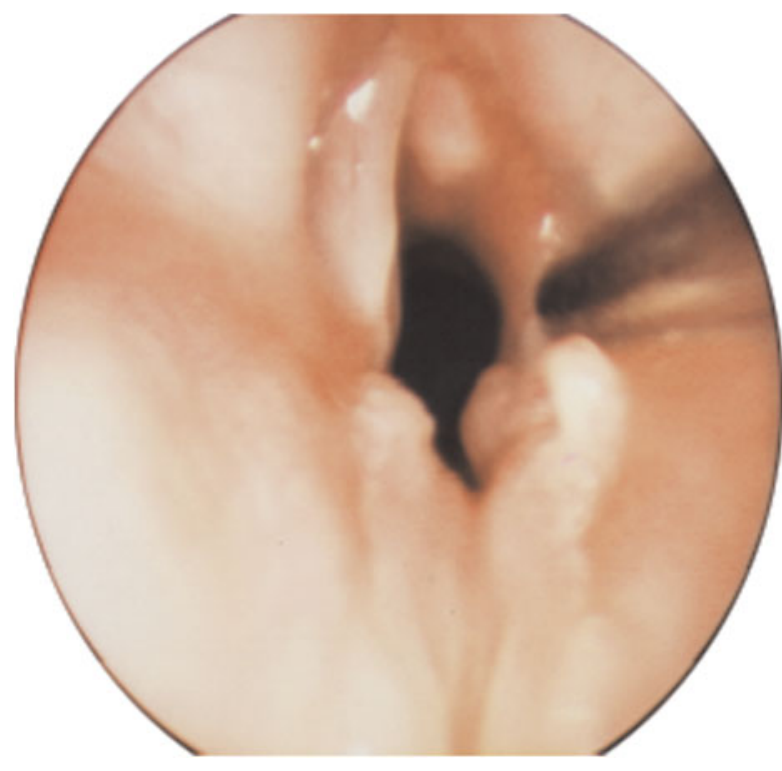

FIG. 4

Case four: Absent middle third right vocal fold.

Microlaryngoscopy at eight months revealed grade one subglottic stenosis and complete absence of the anterior two-thirds of the left vocal fold (Figure 5). The right vocal fold was normal. At such a young age, it was difficult to accurately assess the long term implications of this injury for the child's voice.

The child was regularly reviewed over a period of two years. Her symptoms of reflux were successfully treated with medication. The strength of her cry was reported to have improved. At 18 months, the child's family felt her voice was still hoarse. At this time, she underwent implantation of a unilateral cochlear implant.

By the age of three years, the child's family were very happy with her voice, which they now felt was normal, and she was discharged to the care of her local speech and language therapy team.

\section{Discussion}

Airway distress at birth can be traumatic for the family and the delivery room team, as well as for the patient. Paediatric airway expertise is often not present, and the decision to

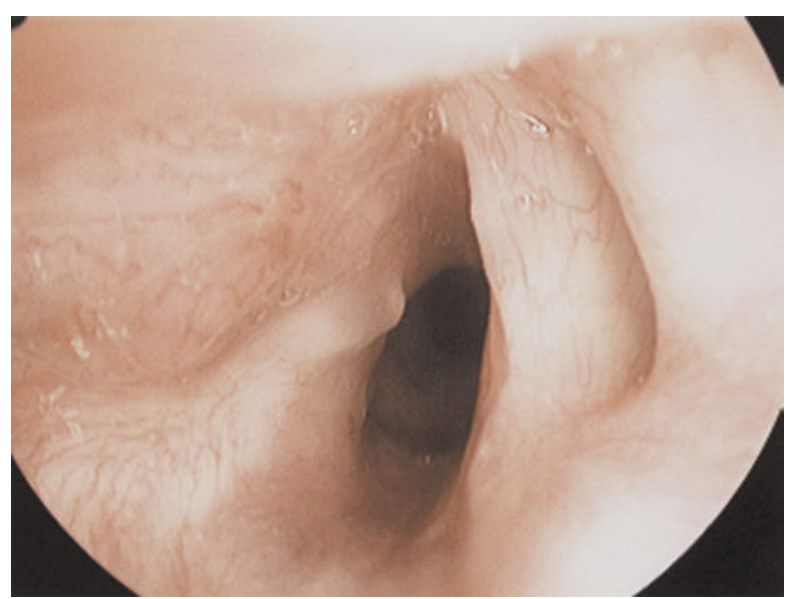

FIG. 5

Case five: Grade 1 subglottic stenosis and absence of anterior two-thirds of left vocal fold. 
undertake early intubation rests with local healthcare professionals on site. ${ }^{2}$ Their decision may have long term sequelae, as neonatal endotracheal intubation is not without complication.

Mucosal tears as a result of intubation are relatively common. Usually, these are superficial and are created when the blade of the laryngoscope injures the vallecula or the supraglottic larynx. The endotracheal tube may cause a tear in the supraglottic larynx, the vocal folds, the lateral wall of the pyriform sinus or the oesophageal inlet. The most minimal supraglottic injury is mucosal erosion of the vocal process of the arytenoids. This injury is symmetrical and will occur even after only a few days of intubation. ${ }^{3}$ Mucosal erosion may lead to the formation of a granuloma if re-epithelialisation fails to cover the mucosal ulcer, after removal of the endotracheal tube.

During prolonged intubation, the endotracheal tube sits between the vocal folds and exerts pressure in lateral and posterior directions. This pressure exceeds the mucosal capillary perfusion pressure, causing ischaemic necrosis in the contact area between the tube and the laryngeal surface. The presence of a nasogastric tube, which may trap the posterior larynx between itself and the endotracheal tube, is a compounding factor in laryngeal injury, as is the size of the endotracheal tube. ${ }^{4}$

After prolonged intubation, some degree of laryngeal injury is noted in virtually every patient examined after extubation. After seven or more days of intubation, all patients can be expected to have erythema of the laryngeal mucosa, and 70 per cent or more will be noted to have ulceration. ${ }^{5}$

In the paediatric age group, and particularly in children requiring intensive care treatment, intubation injury is most likely to occur at the level of the subglottic larynx, because the cricoid ring is relatively smaller than the glottis at this stage of development. ${ }^{4}$ The newborn vocal fold is thick relative to its length and does not contain a discrete ligamentous structure. The mucosal lamina propria is nearly uniform in structure, resembling the superficial layer of the lamina propria in adults. The vocal folds have an excellent blood supply carried by paired anterior commissure branches from the cricothyroid arcade, which originate from the anterior division of the superior laryngeal artery. ${ }^{6}$

The treatment of vocal fold injury involves the whole multidisciplinary team, and includes medical, surgical and voice therapy management. Voice management begins early in the rehabilitative process and continues throughout treatment. Voice assessment is conducted using a formal scheme (e.g. the Vocal Profile Analysis Protocol) ${ }^{7}$ modified to account for some of the additional features found in this patient group, including supraglottic voice. Instrumental analysis (e.g. electrolaryngography) ${ }^{2}$ helps clarify perceptual impressions of voice (e.g. regarding pitch and quality), but also provides additional diagnostic information (e.g. contact quotients) which can guide diagnosis and therapy. Voice clinic assessment with dynamic laryngoscopy and stroboscopy provides clarification of both the movement potential of the intact vocal fold and the compensatory mechanisms involved, and thus informs management planning and patient education.

Early interventions include programmes that promote approximation of the intact vocal fold to residual tissues at the glottic level, using vocal play. At this stage, the development of supraglottic voice is not promoted, but may be so later if the voice does not develop from structures at the glottic level. In the authors' experience, nearly all children can engage in direct voice therapy interventions (e.g. modification of breathing patterns and muscle tension) from a developmental age of eight years. A smaller number can tackle these abstract voice concepts earlier, and candidacy for voice therapy must therefore be determined at an individual level. Voice therapy can result in improved frequency range, strength, control, agility and quality of voice. $^{8}$

Vocal features will vary according to the injury and whether or not the child attempts to compensate with vocal tension behaviours. Whilst the attainment of normal voice is not realistic, speech intelligibility can be greatly improved by preventing or correcting negative vocal gestures (e.g. inspiratory voice and supraglottic phonation) and vocal effort. The child may be suitable for augmentative communication systems such as a voice amplifier, but careful assessment is necessary. The vocal impairment can be considerable, and the psychosocial impact on the child and adolescent should not be underestimated, although there is considerable variation amongst patients. It is important to evaluate this impact and to support the child and family as needs change with maturation.

Therapy in these children may be complicated by additional problems associated with prematurity (e.g. learning or hearing difficulties.)

Medical management includes treatment with steroids and antibiotics, especially when signs of inflammation exist; treatment for gastroesophageal reflux is also used. Surgical management usually follows a period of medical management and voice therapy, although cases should be carefully selected.

Hartnick et al. ${ }^{9}$ described the development and maturation of the paediatric vocal fold lamina propria, using 34 archived paediatric larynges ranging from newborn to 18 years. Many of the contemporary phonosurgical procedures are subepithelial dissections and, as such, spare the superficial lamina propria. It is difficult to define the appropriate age at which these 'adult' procedures can be applied to the paediatric vocal fold. Hartnick and colleagues' data suggest that, in appropriately selected patients in whom pre-, peri- and post-operative voice therapy is possible, phonosurgical excisions can be accomplished by means of subepithelial flap elevation and superficial lamina propria preservation, at ages as young as seven years.

- This paper reports five cases of neonatal laryngeal injury affecting the paediatric voice

- Significant voice disorder secondary to vocal fold injury may be a long term complication of neonatal intubation

- Such children require follow up throughout childhood and beyond puberty as their vocal needs and abilities change

- Regular specialist voice therapy evaluation is recommended, with consideration of phonosurgical techniques including injection laryngoplasty and thyroplasty

The goals of surgery are to optimise glottic closure and mucosal wave function. For mucosal tears without tissue deficit, immobilisation and adequate apposition of the injured vocal fold for $48-72$ hours provides adequate time for angiogenic growth and early wound healing. Wohl ${ }^{10}$ achieved a good result in this manner with an uncuffed endotracheal tube and three days of paralysis with sedation. The uncuffed tube causes minimal tissue trauma upon extubation, in contrast to the potential for a cuffed tube to dislodge loosely attached tissue. Surgical options to correct glottic closure include thyroplasty, ${ }^{11}$ autologous fat injection ${ }^{12,13}$ and collagen injection, ${ }^{14}$ amongst other techniques. 
A stiff, adynamic mucosal wave may be improved with collagen injection, ${ }^{14}$ flaps or grafts, ${ }^{15}$ and/or autologous fat implantation. ${ }^{16}$ Mikaelian et al. reported encouraging results for fat implantation via lateral injection into the thyroarytenoid muscle, to medialise and augment paralysed vocal folds. ${ }^{12}$ Brandenburg et al. treated 12 patients with lateral fat injection, and obtained long-lasting improvement of glottic insufficiency via medial displacement and addition of bulk to the vocal fold. ${ }^{13}$ Chan and Titze ${ }^{15}$ showed that fat may be a better implantation material than Gelfoam or collagen because of its viscoelastic properties, which are closest to normal mucosa. ${ }^{17}$ Furthermore, fat autografts have demonstrated tissue stability and long term viability ${ }^{8}$ however, survival may depend upon purification and harvesting techniques. ${ }^{18}$ As some fat is resorbed, laterally placed fat should be overinjected by about 30 per cent.

\section{Conclusion}

This paper reports five cases of neonatal laryngeal injury affecting the paediatric voice. Significant voice disorder secondary to vocal fold injury may be a long term complication of neonatal intubation. The degree of laryngeal injury may not always be a predictor of the degree of dysphonia, as shown in our first case. Such children require follow up throughout childhood and beyond puberty as their vocal needs and abilities change. At the time of writing, none of the patients in the current series had yet undergone reconstructive or medialisation surgery. However, regular specialist voice therapy evaluation is recommended, with consideration of phonosurgical techniques including injection laryngoplasty and thyroplasty.

\section{References}

1 Jesudason W, Rothera MP. An unusual pattern of intubation injury? J Laryngol Otol 2003;117:145

2 Nakayama DK, Gardner MJ, Rowe MI. Emergency endotracheal intubation in paediatric trauma. Ann Surgery 1990;211:218-23

3 Whited R. A study of post-intubation laryngeal dysfunction. Laryngoscope 1985;95:727-9

4 Weymuller E. Prevention and management of intubation injury of the larynx and trachea. Am J Otolaryngology 1992:13:139-44
5 Santos P, Alfrassiabi A, Weymuller E. Risk factors associated with prolonged intubation and laryngeal injury. Otolaryngol Head Neck Surg 1994;111:453-9

6 Pearson BW. Laryngeal microcirculation and pathways of cancer spread. Laryngoscope 1975;85:700-13

7 Laver J. The Phonetic Description of Voice Quality. Cambridge: Cambridge University Press, 1980

8 Benninger MS, Alessi D, Archer S, Bastian R, Ford C, Kouffman $\mathrm{J}$ et al. Vocal fold scarring: current concepts and management. Otolaryngol Head Neck Surg 1996;115: $474-82$

9 Hartnick CJ, Rehbar R, Prasad V. Development and maturation of the paediatric human vocal fold lamina propria. Laryngoscope 2005;115:4-15

10 Wohl D. Traumatic vocal fold avulsion injury in a newborn. $J$ Voice 1996;10:106-8

11 Maves MD, McCabe BF, Gray S. Phonosurgery: indications and pitfalls. Ann Otol Rhinol Laryngol 1989;98:577-80

12 Mikaelian D, Lowry LD, Sataloff RT. Lipoinjection for unilateral vocal cord paralysis. Laryngoscope 1991;101:465-8

13 Brandenburg JH, Kirkham W, Koschkee D. Vocal cord augmentation with autogenous fat. Laryngoscope 1992;102: 495-500

14 Ford CN, Bless DM, Loftus JM. Role of injectable collagen in the treatment of glottic insufficiency. A study of 119 patients. Ann Otol Rhinol Laryngol 1992;101:237-47

15 Bouchayer M, Cornut G. Microsurgery for benign lesions of the vocal folds. Ear Nose Throat J 1988;11:446-66

16 Sataloff RT, Spiegel JP, Hawkshaw M, Rosen DC, Heuer RJ. Autologous fat implantation for vocal fold scar: a preliminary report. J Voice 1997;11:238-46

17 Chan RW, Titze IR. Viscosities of implantable biomaterials in vocal fold augmentation surgery. Laryngoscope 1998; 108:725-31

18 Mikus JL, Koufman JA, Kilpatrick SE. Fate of liposuctioned and purified autologous fat injections in the canine vocal fold. Laryngoscope 1995;105:871-4

Address for correspondence:

Mr D Bray,

6 Bedford Terrace,

Royal Tunbridge Wells TN1 1YJ, UK.

E-mail: dbray@doctors.org.uk

Mr D Bray takes responsibility for the integrity

of the content of the paper.

Competing interests: None declared 